\title{
EFFICIENT MOTION ESTIMATION USING SORTING-BASED PARTIAL DISTORTION SEARCH
}

\author{
Chou-Chen Wang, Chia-Jung Lo and Cheng-Wei Yu \\ Department of Electronic Engineering, \\ I-Shou University, Kaohsiung, Taiwan \\ E-mail: chchwang@isu.edu.tw
}

\begin{abstract}
An efficient motion-estimation algorithm based on partial block distortion using sorted significant features including bit-plane and mean is proposed. The proposed algorithm can obtain relatively accurate motion vectors with a reduced computational load. Simulation results show that the proposed method achieves its MSE performance very close to the full search method, while requiring only $6-8 \%$ of the computation needed by the full search. Furthermore, the performance of our method is better than other algorithms based on partial block distortion search.
\end{abstract}

\section{INTRODUCTION}

Motion compensation is a key component of many videocoding standards (e.g., ISO MPEG-1/2/4 and ITU-T H.261/263/264) due to its high efficiency in reducing temporal redundancy between successive frames and its implementation simplicity. Block-based motion estimation is the most popular method to obtain motion-compensated prediction. By dividing each frame into rectangular blocks of equal size, the motion estimator obtains a motion vector (MV) for each of the blocks within a search window in the reference frame using the block matching algorithm (BMA). The full search algorithm (FSA) is the most straightforward BMA, which provides an optimal solution by matching all the candidate blocks inside a search window. However, the computational complexity of FSA is always too high for real-time implementation. Therefore, many fast motion estimation algorithms to reduce the computation of the FSA have been proposed over the past few decades [1-8]. These fast motion estimation using BMAs can be classified into two groups. One is the lossy motion estimation algorithm, and the other is the lossless one. The lossy motion estimation (e.g., NTSS [1], DS [2] and HEXBS [3]) has some degradation of predicted images compared with the conventional FSA, and the lossless motion estimation obtains no degradation of predicted images compared with the conventional FSA (e.g., SEA [4], PDE [5] and ASSA
[6]). Recently, the improved versions [7-9] for lossy motion estimation using partial distortion elimination algorithm (PDE) have been proposed for more accurate prediction. Among these improved methods, the normalized partial distortion search (NPDS) proposed by Cheung and Po [7] is a good approach in reducing computational burden. However, a problem of them is that the searching speed and its MSE performance cannot be still satisfied in practical applications. Therefore, many methods based on the NPDS are proposed to further improve the searching efficiency of the NPDS [8-9]. These methods mainly focus on the image complexity of an image block and propose adaptive matching scan algorithms to determine the matching order by sorting gradient magnitude of sub-blocks in a macro block (MB) of the current frame in descending order [1314]. However, a high overhead and MSE degradation exist in these methods when compared with FSA.

In this paper, we suggest further technique to improve the searching efficiency of the NPDS. We propose an efficient NPDS algorithm by using the characteristics of patterns similarity. Two significant features of image block pattern including bit-plane and mean are used in the proposed method. The proposed approach is significantly more efficient as compared to the algorithm [8] which makes use of pixel gradient properties in an adaptive NPDS.

\section{OVERVIEW OF NPDS}

In this section, we briefly overview the NPDS algorithm since it is the basis of our new algorithm. The motion estimation process is to obtain a MV for a target MB by using the block matching technique, which minimizes a measure of matching distortion between the target $\mathrm{MB}$ in the current frame and a candidate $\mathrm{MB}$ within a search window in a reference frame. The displacement between the candidate $\mathrm{MB}$ with the smallest distortion and the target $\mathrm{MB}$ will be selected as the resulting MV. One of the most frequently used criteria to measure the matching distortion is the sum of absolute difference (SAD). The SAD between a target $\mathrm{MB}$ at position $(x, y)$ in the current frame, $I_{t}$, and a candidate $\mathrm{MB}$ at position $(x+u, y+v)$ in the reference 


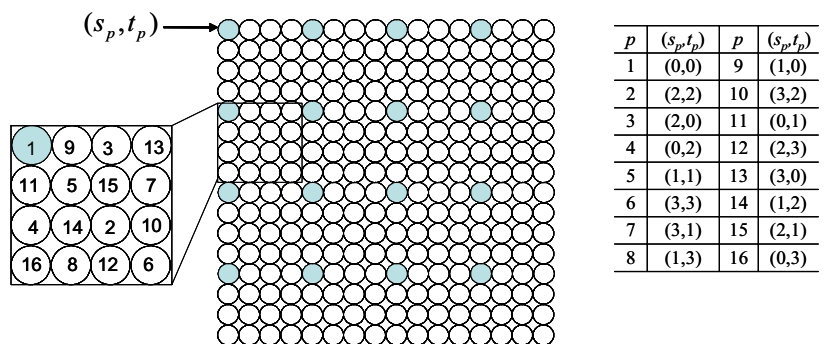

Fig.1 The locations for the calculation order of the partial distortion. $\left(s_{p}, t_{p}\right)$ is the offset of the upper left corner point of the $p$ th partial distortion.

frame, $I_{t-1}$, is defined as follows:

$$
\begin{aligned}
D(x, y ; u, v)=\sum_{i=0}^{15} \sum_{j=0}^{15} \mid I_{t}(x+i, y+j)- \\
\quad I_{t-1}(x+i+u, y+j+v) \mid
\end{aligned}
$$

where $16 \times 16$ is size of a $\mathrm{MB} ; I_{t}(\cdot, \cdot)$ and $I_{t-1}(\cdot, \cdot)$ represent pixels intensity in the current frame and the reference frame, respectively. Based on halfway-stop idea, the partial distortion of the NPDS is defined as a group of pixels' distortions instead of a single pixel's distortion. Thus, the block distortion $D(x, y ; u, v)$ is divided into 16 partial distortions $\left(d_{p}\right)$, where each partial distortion consists of 16 points spaced equally between adjacent points, as shown in Fig. 1. The $p$ th partial distortion is defined as

$$
\begin{aligned}
d_{p}(x, y ; u, v) & =\sum_{i=0}^{3} \sum_{j=0}^{3} \mid I_{t}\left(x+4 i+s_{p}, y+4 j+t_{p}\right) \\
& -I_{t-1}\left(x+4 i+s_{p}+u, y+4 j+t_{p}+v\right) \mid
\end{aligned}
$$

where $\left(s_{p}, t_{p}\right)$ is the offset of the upper left corner point of the $p$ th partial distortion from the upper left corner point of the candidate block. The order of calculation of $d_{p}$ is depicted in the left part of Fig. 1. The $p$ th accumulated partial distortion is defined as

$$
D_{p}(x, y ; u, v)=\sum_{i=1}^{p} d_{i}(x, y ; u, v)
$$

The NPDS matches all the search points inside the search window as that in the FSA. The search begins at the origin search point and moves outward with a spiral scanning path. During each block matching, the NPDS compares each accumulated partial distortion $D_{p}$ with the normalized minimum distortion (NMD: $p D_{\min } / 16$ ) where $D_{\min }$ is the current minimum distortion. The comparison starts from $p=1$ and proceeds toward $p=16$, and the comparison is stopped if the normalized partial distortion of the candidate motion vectors (CMVs) is greater than the NMD. At the end of comparison (i.e. $p=16$ ), if $D_{16}$ is smaller than $D_{\min }$, then this CMV becomes the new current minimum point. By comparing the normalized partial distortion against the NMD, computational complexity is reduced by high rejection of impossible CMVs at early stage.

\section{THE PROPOSED METHOD}

From the above discussions of NPDS, we can find the reduction of distortion calculation in obtaining a MV with the PDE algorithm depends on how fast global minimum of matching distortion is detected. If we find the global minimum of distortion in the calculation of the matching error faster, then the complete computation of the matching error in a block is avoided and partial SAD is determined faster. However, the NPDS uses the evenly dithering order (see Fig. 1) which takes account of pixels being evenly distributed on the block. Therefore, the speedup ratio to find MVs is limited due to uniform calculation order. It seems attractive to further reduce the computation complexity by appropriately arranging the partial SAD calculation order according to some significant features.

In this paper, we make use of the fact that the blockmatching error between the current block and the reference block is proportional to the pattern similarity. The motivations of the proposed algorithm are using two significant features of bit-plane and mean for sub-blocks in a MB to find the impossible candidates faster. With the above-observation, we propose a two-stage matching scan algorithm for more computational reduction than that of the previous works [7-8].

In the first stage, a binary bit-plane is derived for each sub-block in a MB to represent its direction and pattern information. Given a sub-block in a MB with size $4 \times 4$, its corresponding bit-plane $B_{p}^{I_{t}}$ in the current frame is simply calculated by

$$
\begin{aligned}
B_{p}^{I_{t}}(x+4 i+ & \left.s_{p}, y+4 j+t_{p}\right)= \\
& \begin{cases}1 & \text { if } I_{t}\left(x+4 i+s_{p}, y+4 j+t_{p}\right) \geq m_{p}^{I_{t}} \\
0 & \text { otherwise }\end{cases}
\end{aligned}
$$

where $i=0,1,2,3, j=0,1,2,3$ and $I_{t}(\cdot, \cdot)$ represents the $\left(x+4 i+s_{p}, y+4 j+t_{p}\right)$ th pixel value of the sub-block, and $m_{p}^{I_{t}}$ represents the $p$ th mean value of the sub-block in the current frame. If a sub-block has a bit-plane that is very different from that of another sub-block, the two blocks are unlikely to have similar image characteristics and result in a high matching error. Based on these characteristics, in the first stage, the Hamming distance based on two bit-planes is used as the measurement of similarity. The Hamming distance $H_{p}$ is defined as

$$
\begin{aligned}
H_{p}= & \sum_{i=0}^{3} \sum_{j=0}^{3} B_{p}^{I_{t}}\left(x+4 i+s_{p}, y+4 j+t_{p}\right) \oplus \\
& B_{p}^{I_{t-1}}\left(x+4 i+s_{p}, y+4 j+t_{p}\right)
\end{aligned}
$$


where $B_{p}^{I_{t-1}}$ denote the bit-plane of the sub-block in the reference frame and $\oplus$ denotes the modulo- 2 addition (XOR logic operation). The Hamming distance for each sub-block in a MB is carried out and the magnitudes are sorted in the descending order. It is noted that the different contrast of pixels intensity in the block maybe have the same directive patterns so that result in the same bit-plane and $H_{p}$. In order to further separate the similarity between two sub-blocks from the same $H_{p}$, an absolute difference of means (ADM) measure is adopted in the second stage. It is defined as

$$
A D M_{p}=\left|m_{p}^{I_{t}}-m_{p}^{I_{t-1}}\right|
$$

where $m_{p}^{I_{t-1}}$ denote the $p$ th mean value of the sub-block in a MB in the reference frame. Then, the ADM magnitudes for the same $H_{p}$ are resorted in the descending order.

The first stage mainly computes directional differences using binary pattern matching for all pixels in the center block of the search window, that is to say the one with the null candidate motion vector. The magnitudes of Hamming distances of sub-blocks in a MB are sorted in descending order. In the second stage, if the sub-blocks have the same Hamming distances, then the ADM magnitudes of these sub-blocks are resorted in descending order. The positions are first sorted in descending order according to our proposed two-stage matching scan method, and then used to determine the order of matching priority for all the candidate blocks in the search window. According to the arranged sub-block, we find the best MV using the same search procedure as the NPDS. The determining process of the partial SAD calculation order is an overhead computation. However, the cost of overhead (only needs an average operation of 720 additions, 32 divisions, 768 comparisons and 4.7 absolutes for a motion vector) can be neglected when compared with the total computational load.

\section{SIMULATION RESULTS}

To compare the performance of the proposed algorithm with the PDE-based search algorithms, we use 80 frames of "foreman," "football," “tennis," "flower," "akio," and "salesman" video sequences. All the concerning BMAs are implemented using Visual $\mathrm{C}++$ and the Pentiumn IV $1.3 \mathrm{G}$ PC. For a fair comparison, the FSA, the NPDS and the gradient-based PDS (GB-PDS) [8] are also carried out under the same conditions. The MB size is $16 \times 16$ pixels, and the search window is $31 \times 31$ pixels. The image format is CIF $(352 \times 288)$ for each sequence, and only forward prediction is used. Overhead computations are also included in our experimental results. The simulation results are shown in terms of the average number of checking subblock with the reference of that of full search per MB and

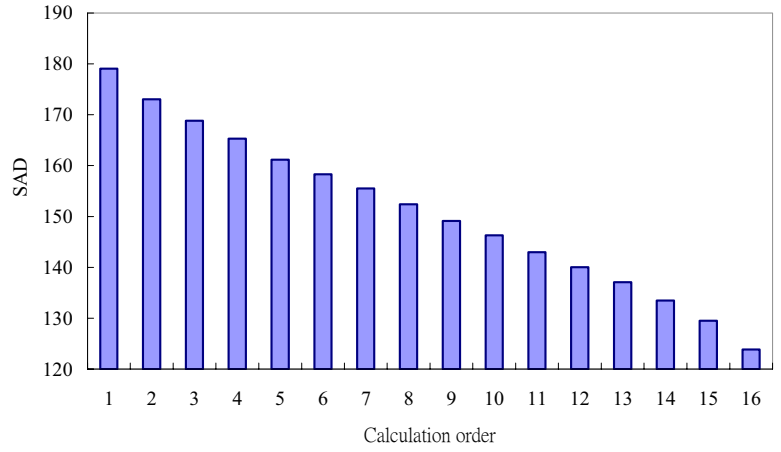

Fig. 2 Average partial distortions for sorted significant subblock using the proposed two-stage matching scan.

MSE performance. In addition, all the matching scan algorithms employed the outward spiral search.

Suppose that the partial distortion of a candidate block properly represents the SAD of the block, we can use it for the block distortion comparison with low computational complexity. In fact, the SAD between two MBs depends on a small number of significant sub-blocks that have higher matching errors. Figure 2 shows that the average partial distortions for sorted significant sub-block are proportional to the calculation order using the proposed two-stage matching scan. In other words, we can find that it is true the lower calculation order the higher matching distortion in our method. The first partial distortions are much larger than those for evenly dithering order.

Figure 3 compare the MSE performance of different BMAs for the "foreman" and "tennis" sequences using the first 40 frames. It is clearly observed that the proposed algorithm can maintain their MSE performances very close to the FSA, while the other fast BMA's produce much higher average MSEs. Table I shows the average computational reduction based on square sub-blocks per MB required by different BMA's. As we predicted, we can find that the proposed method can further reduce the computational load than the evenly dithering order matching scan algorithm (NPDS) and the complexity ordered matching scan algorithm (GB-PDS). In Table I, we can see that the proposed method requires only $6-8 \%$ of the computation needed by the FSA.

\section{CONCLUSIONS}

In this paper, we have shown the relationship between matching error and significant features (bit-plane and mean) for image block and proposed the excellence of matching scan for computational reduction. By using the calculation order according to pattern similarity, we can obtain faster elimination of impossible candidate vectors than other PDEbased algorithms. 


\section{REFERENCES}

[1] R. Li, B. Zeng, and M. L. Liou, "A new three-step search algorithm for block motion estimation," IEEE Trans. Circuits Syst. Video Technol., vol. 4, no. 5, pp. 438-442, Aug. 1994.

[2] S. Zhu and K. K. Ma, "A new diamond search algorithm for fast block-matching motion estimation," IEEE Trans. Image Processing, vol. 9, pp. 287-290, Feb. 2000.

[3] C. Zhu, X. Lin, and L. P. Chau, "Hexagon-based search pattern for fast block motion estimation," IEEE Trans. Circuits Syst. Video Technol., vol. 12, pp. 349-355, May 2002.

[4] W. Li and E. Salari, "Successive elimination algorithm for motion estimation," IEEE Trans. Image Process., vol. 4, no. 1, pp. 105-107, Jan. 1995.

[5] S. Eckart and C. Fogg, "ISO/IEC MPEG-2 software video codec," Proc. SPIE, vol. 2419, pp. 100-118, 1995.

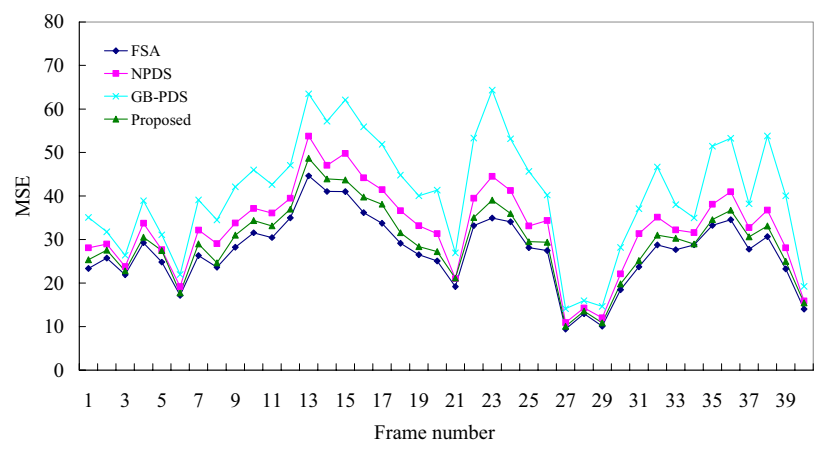

(a)
[6] B. Liu and A. Zaccarin, "New fast algorithms for the estimation of block motion vectors," IEEE Trans. Circuits Syst. Video Technol., vol. 3, pp. 148-157, Apr. 1993.

[7] C. K. Cheung and L. M. Po, "Normalized partial distortion search algorithm for block motion estimation," IEEE Trans. Circuits Syst. Video Technol., vol. 10, no. 3, pp. 417-422, Apr. 2000.

[8] J. N. Kim, S. C. Byun, Y. H. Kim, and B. H. Ahn, "Fast full search motion estimation algorithm using early detection of impossible candidate vectors," IEEE Trans. Signal Process., vol. 50, no. 9, pp. 2355-2365, Sep. 2002.

[9] B. Montrucchio and D. Quaglia, "New sorting-based lossless motion estimation algorithms and a partial distortion elimination performance analysis," IEEE Trans. Circuits Syst. Video Technol., vol. 15, no. 2, pp. 210-220, Feb. 2005.

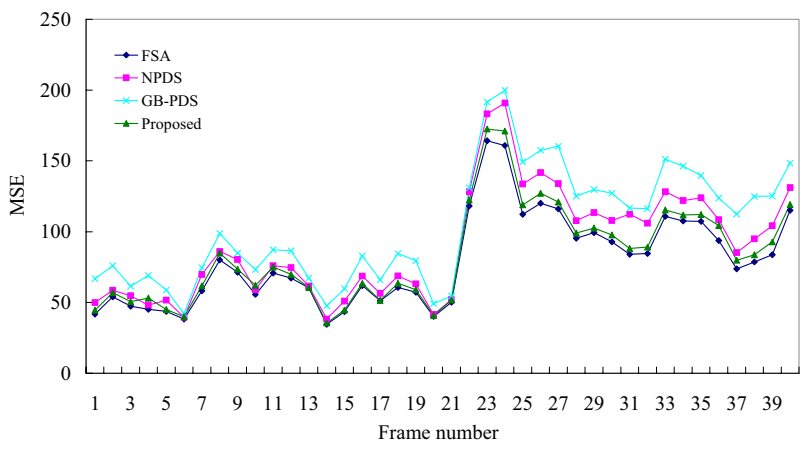

(b)

Fig. 3 Compare the MSE performance of different BMAs (a) "foreman" sequence (b) "tennis" sequence.

Table I. Average checking sub-block per MB required by different fast algorithms with search window $31 \times 31$ in 30 frames/s.

\begin{tabular}{|c|c|c|c|c|c|c|}
\hline $\begin{array}{c}\text { Sequences } \\
\text { Methods }\end{array}$ & Foreman & Akio & Tennis & Salesman & Flower & Football \\
\hline FSA & 16 & 16 & 16 & 16 & 16 & 16 \\
\hline NPDS & 1.264 & 1.467 & 1.258 & 1.1 & 1.39 & 1.6 \\
\hline GB-PDS & 1.049 & 1.018 & 1.077 & 1.027 & 1.053 & 1.441 \\
\hline Proposed & 1.028 & 1.001 & 1.037 & 1.011 & 1.092 & 1.254 \\
\hline
\end{tabular}

